Revue Gouvernance

Governance Review

GOUVERNANCE

(G) SRNAWCL

\title{
L'exercice délibératif dans le contexte de rapports linguistiques complexes : le cas du Nouveau-Brunswick
}

\section{Christine C. Paulin}

Volume 14, numéro 1, 2017

URI : https://id.erudit.org/iderudit/1040635ar

DOI : https://doi.org/10.7202/1040635ar

Aller au sommaire du numéro

Éditeur(s)

Centre d'études en gouvernance de l'Université d'Ottawa

ISSN

1912-0362 (numérique)

Découvrir la revue

Citer cet article

Paulin, C. C. (2017). L'exercice délibératif dans le contexte de rapports

linguistiques complexes : le cas du Nouveau-Brunswick. Revue Gouvernance /

Governance Review, 14(1), 20-42. https://doi.org/10.7202/1040635ar

\section{Résumé de l'article}

Dans la littérature sur les instruments d'action publique (IAP), il n'y a que très peu de prise en considération des variables contextuelles et de leurs effets sur la conception des instruments, de même que sur le processus de formulation des politiques publiques. Or, par l'entremise d'une étude empirique sur la question à partir du cas du Nouveau-Brunswick, nous pouvons mettre en relief le rôle de la dimension linguistique en tant que l'une des variables contextuelles ayant le plus d'influence sur la conception des IAP (plus précisément sur les mécanismes délibératifs) et sur le processus décisionnel qui en découle. En particulier, nous observons l'incidence que peut avoir la variable linguistique sur les mécanismes délibératifs, mais également sur les réseaux d'action publique impliqués dans l'exercice démocratique, sur les institutions étatiques, et sur le processus de production des politiques publiques. Nos constats permettent de conclure que les instruments se traduisent différemment dans un contexte de rapports linguistiques complexes (un bilan qui peut d'ailleurs se transposer à l'infranational bilingue, où cohabitent diverses communautés linguistiques). 


\title{
L'exercice délibératif dans le contexte de rapports linguistiques complexes : Le cas du Nouveau- Brunswick
}

\author{
Par Christine C. Paulin ${ }^{i}$
}

\begin{abstract}
Résumé
Dans la littérature sur les instruments d'action publique (IAP), il n'y a que très peu de prise en considération des variables contextuelles et de leurs effets sur la conception des instruments, de même que sur le processus de formulation des politiques publiques. Or, par l'entremise d'une étude empirique sur la question à partir du cas du NouveauBrunswick, nous pouvons mettre en relief le rôle de la dimension linguistique en tant que l'une des variables contextuelles ayant le plus d'influence sur la conception des IAP (plus précisément sur les mécanismes délibératifs) et sur le processus décisionnel qui en découle. En particulier, nous observons l'incidence que peut avoir la variable linguistique sur les mécanismes délibératifs, mais également sur les réseaux d'action publique impliqués dans l'exercice démocratique, sur les institutions étatiques, et sur le processus de production des politiques publiques. Nos constats permettent de conclure que les instruments se traduisent différemment dans un contexte de rapports linguistiques complexes (un bilan qui peut d'ailleurs se transposer à l'infranational bilingue, où cohabitent diverses communautés linguistiques).
\end{abstract}

Mots clés : Démocratie délibérative, instruments d'action publique, variable linguistique, réseaux d'action publique, institutions étatiques et processus de production des politiques publiques

\begin{abstract}
In the literature on public policy instruments, there is very little consideration of contextual factors and their effects on the design of policy instruments, as well as on the decision-making process. An empirical study of the situation in New Brunswick, allows to identify the linguistic dimension as one of the most influential contextual factor in the design of public policy instruments (namely called deliberative mechanisms) and the resulting decision-making process. In this sense, we observe the impact of the linguistic variable at the level of deliberative mechanisms, but also at the level of the networks involved in the democratic exercise, at the level of state institutions, and at the level of the decision-making process. Our findings make it possible to conclude that public policy tools are translated differently in the context of complex linguistic relations (an Revue Gouvernance

Volume 14, numéro 1, 2017


assessment that can be transposed to the bilingual subnational context where various linguistic communities coexist).

Key words: deliberative democracy, public policy instruments, linguistic variable, public policy networks, state institutions and decision-making process

\section{Introduction}

En réponse au besoin d'une plus grande participation des citoyens dans le processus d'élaboration des politiques publiques, certains auteurs considèrent les mécanismes délibératifs comme une option viable pour compléter, voire remplacer les consultations traditionnelles où le pouvoir décisionnel est généralement exclusivement confié aux responsables politiques et aux experts (Arnstein, 1969). Selon de nombreux auteurs (Black et coll., 2014; Perote-Peña et Piggins, 2015; Steiner, 2012), la conception et la mise en œuvre de ces dispositifs contribuent à renforcer ce qu'ils appellent une «démocratie délibérative ", c'est-à-dire un modèle où la participation des citoyens est centrale dans le processus décisionnel et vise à contrer le déficit démocratique légué par les systèmes représentatifs et l'évolution de nos sociétés modernes. Or, aux côtés de cette perspective idéaliste, une perspective critique des mécanismes délibératifs (Korthals, 2011; Prades, 2006; Ryfes, 2005; Sanders, 1997) souligne les limites de la nouvelle forme de démocratie contemporaine, en pointant du doigt le présupposé caractère inclusif et démocratique des instruments, ainsi que les conditions rationnelles qui les sous-tendent et qui sont considérées comme inatteignables.

Même si les études dans ce domaine ne font pas l'unanimité et qu'elles polarisent le débat, nous ne pouvons ignorer leur pertinence pour comprendre le processus d'élaboration des politiques publiques. En effet, ce sujet entourant les mécanismes délibératifs a fait couler beaucoup d'encre au cours des dernières années et de nombreux débats sur la question ont considérablement participé à l'avancement des connaissances.

Notre questionnement s'inscrit en continuité avec les travaux qui s'intéressent aux effets des facteurs contextuels sur l'ingénierie des instruments et le processus de formulation des politiques publiques. Nos observations rejoignent la perspective dite « classique » des instruments, c'est-à-dire les travaux anglo-saxons menés notamment par Salomon et Lund (1989), Lowi (1985), Hood (1983, 2006, 2007), Howlett (2005, 2011, 2014) et Varone (1998), qui soulignent d'entrée de jeu la technicité (la nature fonctionnelle) de ces dispositifs. 
La littérature fait cependant peu de place à la compréhension et à l'articulation des variables contextuelles inhérentes aux mécanismes délibératifs, ou à leur incidence sur le processus de formulation des politiques publiques (Abelson et Gauvin, 2006; Abelson et coll., 2010; O'Meally, 2013). Or, dans une étude empirique qui met en relief le statut bilingue du Nouveau-Brunswick, nous avons pu constater le rôle de la dimension linguistique en tant que variable contextuelle ayant une forte influence sur le design des mécanismes délibératifs et le processus de formulation des politiques publiques. La question principale qui guide cet article concerne précisément cet aspect: en quoi la dimension linguistique influence-t-elle l'ingénierie des mécanismes délibératifs dans un contexte de rapports linguistiques complexes comme celui du Nouveau-Brunswick?

Pour répondre à cette interrogation, nous analysons l'influence de la variable linguistique sur les mécanismes délibératifs à l'étude, mais également sur les réseaux d'action publique impliqués dans l'exercice démocratique, sur les institutions étatiques et sur le processus de production des politiques publiques. Deux cas ont été analysés : celui de l'élaboration d'un plan de réduction de la pauvreté et celui de l'élaboration d'une politique familiale. Ces derniers partagent plusieurs points communs: ces exercices démocratiques ont eu lieu à la même période temporelle (soit entre 2009 et 2010, au Nouveau-Brunswick), l'enjeu central aborde une problématique sociale et le même mécanisme délibératif a été utilisé pour mener la démarche, soit le Dialogue public. Ils partagent également la particularité d'avoir été conçus et mis en œuvre dans la seule province canadienne à caractère bilingue, où cohabitent deux langues officiellement reconnues par la Loi sur les langues officielles du Nouveau-Brunswick (LLO), soit le français et l'anglais. Par ailleurs, ces cas divergent aussi sur deux points. Premièrement, alors que Le Dialogue sur la réduction de la pauvreté a été conçu et mis en œuvre par des acteurs gouvernementaux, Le Dialogue sur l'élaboration d'une politique familiale a été lancé à l'instigation d'un groupe d'acteurs non gouvernementaux (organismes communautaires ${ }^{1}$ et experts). Deuxièmement, le premier cas a mené à la mise sur pied d'une stratégie provinciale concrète de réduction de la pauvreté, alors que le second n'a jamais donné de résultats tangibles en matière de politique publique. À ce jour, une politique familiale se fait toujours attendre au Nouveau-Brunswick. Mentionnons enfin que les données analysées proviennent de sources secondaires (incluant rapports gouvernementaux, revue de presse, procès-verbaux, document de travail, etc.), de notes de terrain (pendant les entretiens) et d'une série de 33 entretiens semi-structurés auprès d'acteurs gouvernementaux (incluant élus et fonctionnaires) et non gouvernementaux

\footnotetext{
${ }^{1}$ Le Réseau de la petite enfance francophone du Nouveau-Brunswick (RPEFNB), alors piloté par l'Association francophone des parents du Nouveau-Brunswick (AFPNB), est l'organisme communautaire qui a été l'instigateur dans cette démarche démocratique. Les acteurs concernés étaient accompagnés par un groupe d'experts sur la question (chercheurs académiques).
} 
(incluant représentants d'organismes communautaires, experts et citoyens) impliqués d'une façon ou d'une autre dans l'un ou l'autre des cas à l'étude.

\section{Les instruments dans un contexte de démocratie délibérative : état de la littérature}

Les nombreux écrits qui exposent les fondements de la démocratie délibérative permettent de conclure qu'il existe une variété d'angles d'analyse. D'abord, certaines recherches abordent la portée théorique (Beste 2013; Blondiaux, 2008; Goodin, 2012; Sintomer, 2011) et rationnelle (Black et coll., 2014; Fishkin, 2009; Nabatchi, 2010; Venne, 2011) qui sous-tend la présence accrue de cette forme démocratique au sein de nos sociétés. Certaines de ces contributions explicitent les fondements théoriques de la démocratie délibérative de manière à en révéler les particularités, alors que d'autres étudient divers cas empiriques où l'utilisation des mécanismes délibératifs est mise en évidence. D'autres recherches portent plutôt sur les aspects rationnels qui expliquent, du moins en partie, les avantages associés à la démocratie délibérative et à l'utilisation de ces mécanismes lors du processus décisionnel.

Tout comme l'a déjà souligné Blondiaux (2004), nous remarquons que la littérature dans ce domaine oppose essentiellement deux pôles pour expliquer et décrire les écoles de pensée liées à la démocratie délibérative : la perspective «idéaliste», qui s'attache à défendre les bienfaits de l'aspect participatif associé à la démocratie délibérative (Black et coll., 2014; Nabatchi et Amsler, 2014; Venne, 2011), et la perspective "ultra-critique », qui consiste à faire valoir les faiblesses de ce modèle démocratique ou à s'opposer catégoriquement à la perspective idéaliste (Korthals, 2011; Prades, 2006; Ryfe, 2005; Sanders, 1997).

Cette littérature aborde diverses contributions dans de multiples champs de politiques publiques. Les cas empiriques concernent majoritairement les domaines de la santé (Abelson et coll., 2010), de la planification urbaine (Shipley et Utz, 2012) et de l'environnement (Gauthier et coll., 2011), mais discutent également de politiques budgétaires (Tellier, 2014), et de nouvelles technologies de l'information et des communications (Santo et coll., 2010).

La diversité des mécanismes de participation constitue un autre angle d'étude. Dans les écrits sur ce sujet, la tradition anglo-saxonne souligne d'entrée de jeu la technicité, c'està-dire la nature fonctionnelle des dispositifs. En ce sens, les partisans de cette perspective (Hood, 2007; Howlett, 2014; Varone, 1998) misent sur la nature pragmatique des instruments selon deux axes, c'est-à-dire soit en interrogeant leur portée pour en 
comprendre les conditions optimales de formulation et de mise en œuvre (Bresser et O'Toole, 1998; Eliadis et coll. 2005; Howlett et coll., 2009; Linder et Peters, 1989), soit en proposant diverses typologies pour catégoriser les instruments à partir de leurs particularités fonctionnelles (Hood, 1983; Lowi, 1985; Salamon et Lund, 1989). Les instruments sont ainsi vus comme des outils qui permettent de régler l'enjeu public dont il est question, ni plus ni moins. Pour reprendre les propos de Howlett et Ramesh (2003, p.194), les tenants de cette école de pensée laissent entendre qu'il est important de sélectionner "the [...] appropriate tools for the job to be done». Selon eux, les diverses classifications des instruments contribuent à faciliter ce choix du «meilleur » dispositif en fonction de la conjoncture.

Ces typologies sur lesquelles s'appuie l'État pour sélectionner le dispositif le mieux adapté à la nature du problème (dans une perspective de maximisation des bénéfices) regroupent les instruments selon divers critères et caractéristiques. Ces éléments permettent d'établir les distinctions entre différents instruments en fonction de la nature de l'intervention gouvernementale, du degré de coercition, des caractéristiques comportementales ou encore selon les fonctions de l'État (Balch, 1980; Bemelmans-Videc et coll., 2003; Hood, 2007; Howlett, 2011; Lowi, 1966; Schneider et Ingram, 1990). En plus $\mathrm{d}^{\prime}$ analyser les instruments de manière technique et rationnelle, ces typologies ont toutes en commun d'être inspirées par la recherche d'une plus grande efficience et d'une optimisation du niveau d'efficacité au moment de leur conception et de leur mise en œuvre.

D'autres recherches dans le domaine de la démocratie délibérative proposent quant à elles des catégorisations fondées sur l'aspect procédural des instruments (Rowe et coll., 2004), ou encore sur l'éventail des résultats à court ou long terme qui peuvent en découler (Briggs, 2008; Carcasson, 2009). Tout comme les études de l'approche anglo-saxonne des instruments, ces recherches proposent un classement des mécanismes par analogie avec une boîte à outils, où chacun des dispositifs qui s'y retrouve répond à une fonction bien précise et devrait être utilisé dans un contexte défini.

Cette littérature présente des travaux qui ont inspiré la classification des mécanismes délibératifs en fonction du niveau d'engagement des citoyens et du pouvoir décisionnel qui leur est conféré dans le cadre du processus d'élaboration des politiques publiques (Arnstein, 1969; Bishop et Davis, 2002; OCDE, 2001; O’Faircheallaigh, 2010; Yankelovich, 1995). En parallèle, certaines organisations contribuent également à l'avancement des connaissances dans le domaine de la démocratie délibérative, plus particulièrement celles qui concernent les études sur les instruments délibératifs. Parmi celles-ci, le continuum développé au début des années 1990 par l'IAP2 (International Association for Public 
Participation) est à ce jour reconnu comme l'un des cadres de référence le plus couramment utilisé pour catégoriser les instruments délibératifs (Bryson et coll., 2013; Gudowsky et Bechtold, 2013; Lee, 2011; Nabatchi et Amsler, 2014; Sheedy et coll., 2008). Le Gouvernement du Nouveau-Brunswick s'est également inspiré de ce modèle pour élaborer sa politique sur l'engagement des citoyens en 2011 (la Citizen Engagement Policy).

Pour les fins de cet article, et de manière à mieux circonscrire et définir notre objet d'étude, il convient de préciser que les deux cas discutés dans la présente étude se situent à l'extrême droite de ce continuum (voir tableau 1). Ainsi, le dispositif à l'étude, communément appelé Dialogue public, représente le quatrième niveau d'engagement (lequel consiste en une forme de «partenariat » entre l'État et les citoyens qui favorise la prise de décision conjointe).

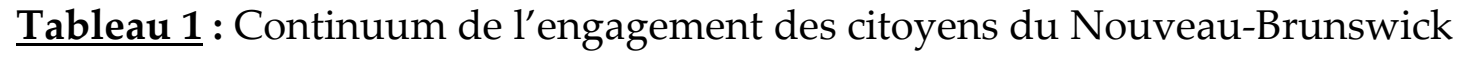

\section{Niveau progressif de la participation des citoyens à la prise de décision}

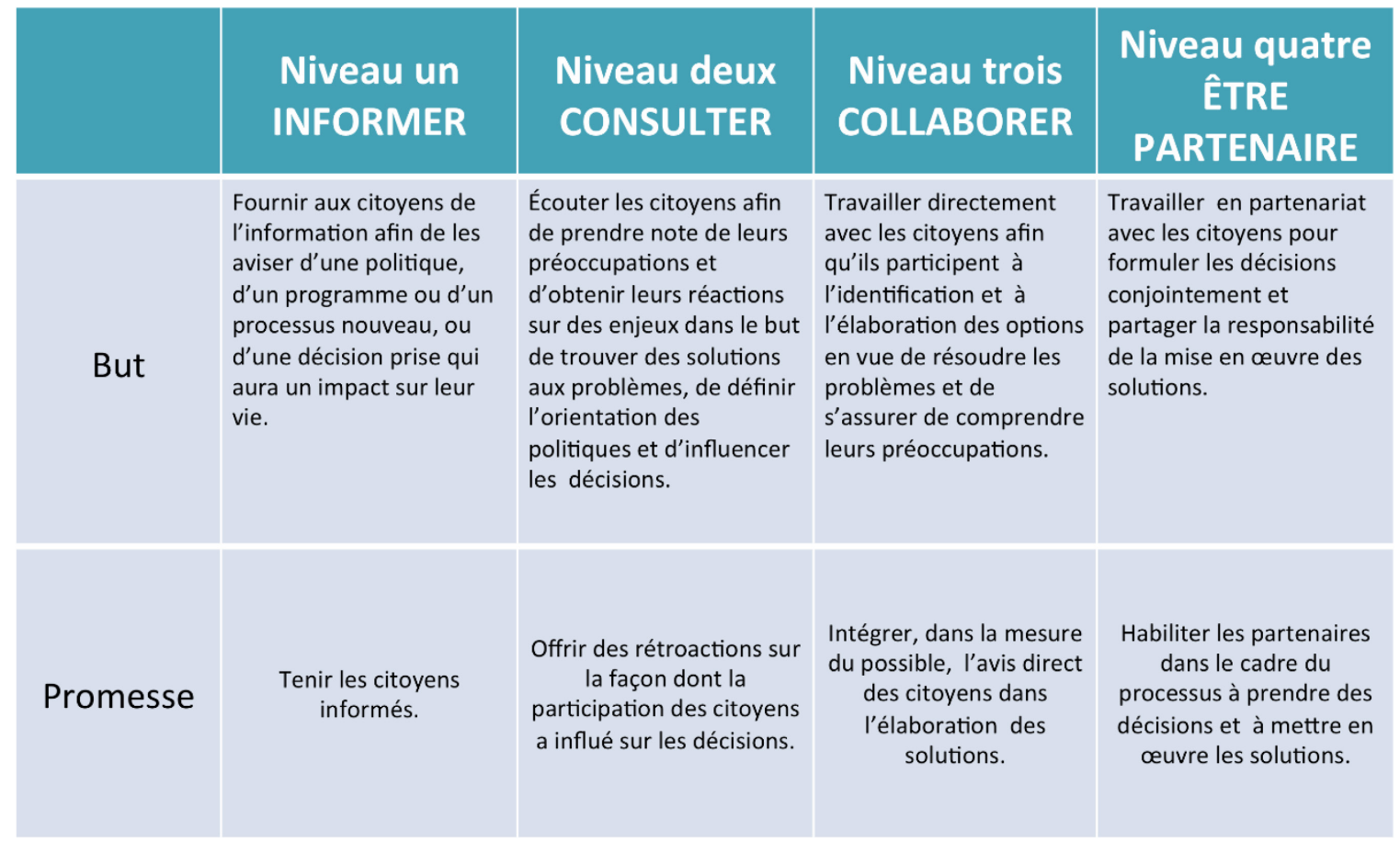

Adapté du Spectrum de la participation du public d'AIP2 et le continuum de participation du public de Santé Canada.

Même si la littérature dans ce champ couvre plusieurs axes de recherche, deux constats se dégagent: peu d'études se concentrent sur la formulation des politiques sociales et 
encore moins suggèrent un cadre d'analyse abordant la jonction entre les facteurs contextuels sous-jacents aux mécanismes délibératifs et leurs effets sur le processus de formulation des politiques publiques (Abelson et coll., 2010; Mitton et coll., 2009; Nabatchi et Amsler, 2014; Thurston, 2005). C'est pourquoi nous proposons d'explorer l'influence de la dimension linguistique en tant que facteur contextuel dominant dans un environnement de rapports sociodémographiques complexes comme celui du NouveauBrunswick, de même que ses effets sur le processus d'élaboration des politiques publiques dans le domaine social. Notre argument principal de recherche aborde précisément cet aspect de la langue, un élément jusque-là ignoré dans la littérature traitant de l'influence des facteurs contextuels sur les instruments.

Selon plusieurs écrits dans ce domaine de recherche, l'environnement externe dans lequel s'insèrent les exercices délibératifs peut considérablement influencer les dispositifs euxmêmes et les politiques publiques qui en découlent (Blondiaux, 2008; McCormack et coll., 2002; Rowe et Frewer, 2000; Sheedy et coll., 2008). Toutefois, les analyses empiriques qui permettent d'établir ces constats et de documenter les leçons apprises ne sont pas suffisamment explicites et trop peu nombreuses. À cet effet, Lee (2011), Ryfe (2007) et Hendriks (2008) précisent que les études qui portent sur l'aspect du design des instruments délibératifs ne présentent pas, ou sinon de manière inadéquate, les particularités du contexte social dans lequel s'inscrivent ces derniers. D'autres travaux, tels que ceux de Bingham et coll. (2005), concluent que d'importantes questions demeurent toujours sans réponses, ou tout au mieux ne sont que partiellement abordées, dans les recherches sur les pratiques de «nouvelle" gouvernance. L'un des angles de recherche qu'ils suggèrent dans ce domaine concerne le lien entre les mécanismes délibératifs en tant qu'exercice démocratique et le processus d'élaboration des politiques publiques : «How does context shape process? Which processes fit best at various stages of the policy cycle? » (Bingham et coll., 2005, p.555).

Mitton et coll. (2009) concluent quant à eux que l'un des principaux constats qui se dégagent des écrits portant sur les spécificités associées au design des mécanismes délibératifs est une invitation aux chercheurs à se pencher sur les éléments pragmatiques qui permettent d'améliorer les résultats qui émanent des exercices délibératifs. Dans le même sens, alors que Beierle et Cayford (2002) suggèrent d'approfondir l'analyse des attributs qui forgent le contexte décisionnel associé à la conception et à la mise en œuvre des instruments délibératifs, d'autres auteurs (Abelson et Gauvin, 2006; Abelson et coll., 2010; Einsiedel, 2002) avancent que l'étude des facteurs contextuels inhérents aux mécanismes délibératifs s'organise autour de deux séries de caractéristiques : celle qui permet de mieux circonscrire l'enjeu public au centre de l'exercice démocratique et celle 
qui caractérise l'organisme responsable de la mise en œuvre du mécanisme (la sponsoring organization).

En nous basant sur le cadre de Abelson et Gauvin (2006), nous retenons les indicateurs suivants, qui s'articulent autour de cinq grands facteurs d'analyse: 1) le contexte sociopolitique, 2) les facteurs communautaires, 3) l'enjeu public, 4) les attributs organisationnels, et 5) le contexte décisionnel (voir figure 1).

Figure 1 : Évaluation des mécanismes délibératifs : modère conceptuel



Source : J. ABELSON et F.-P. GAUVIN (2006). Assessing the Impacts of Public Participation: Concepts, Evidence, and Policy Implications, Canadian Policy Research Networks, page 18.

Le contexte sociopolitique comprend les divers types de relations qui existent entre plusieurs acteurs impliqués dans les processus délibératifs à l'étude, de même que la nature du climat politique qui prévaut dans un secteur de politique publique donné. Les facteurs communautaires englobent les caractéristiques, rôles et influences des citoyens ayant participé aux exercices délibératifs à l'étude pour avoir leur mot à dire sur le processus de production des politiques sociales, ainsi que les dimensions et la nature de l'engagement civique et politique de ces derniers. La nature de l'enjeu public renvoie à son degré d'incertitude scientifique, à sa complexité et à l'information nécessaire à sa 
compréhension par les participants présents à l'exercice délibératif. Le quatrième axe d'analyse, celui des attributs organisationnels, englobe les caractéristiques, rôles et influences des acteurs gouvernementaux impliqués dans l'élaboration de l'exercice délibératif, les pratiques et priorités institutionnelles, de même que le niveau d'intérêt et l'ampleur de l'engagement à l'égard de ce processus délibératif. Finalement, l'analyse du contexte décisionnel aborde les étapes entourant la démarche décisionnelle et l'échéancier des activités.

Dans la deuxième section de cet article, nous présentons les principaux résultats issus des observations empiriques, en portant une attention particulière à la dimension linguistique et à son influence sur la configuration des mécanismes délibératifs et le processus de formulation des politiques publiques qui en découle.

\section{Le quadruple apport de la dimension linguistique dans la compréhension des instruments délibératifs}

L'analyse des données permet d'identifier la dimension linguistique du contexte, qui, à son tour, permet de discuter des apports et limites de l'exercice délibératif de quatre façons : 1) en proposant une typologie des instruments selon le critère linguistique ;2) en dressant un portrait de l'influence de la langue sur les structures et le fonctionnement des groupes/réseaux d'action publique impliqués dans l'exercice délibératif; 3) en cernant le fonctionnement des institutions étatiques dans un contexte de rapports linguistiques complexes; et 4) en décrivant le processus de formulation des politiques publiques au sein d'une province bilingue.

\subsection{La langue comme critère de la typologie des instruments}

La réalité bilingue de la province du Nouveau-Brunswick nous amène à faire le constat que ce contexte particulier influence fortement le design des instruments et, plus largement, le processus de formulation des politiques publiques. À la lumière de notre analyse, trois dispositifs délibératifs se distinguent d'après leur dimension linguistique :

1) les instruments délibératifs dits «bilingues » (avec traduction simultanée);

2) les instruments délibératifs exclusivement en français ou exclusivement en anglais, où participent les citoyens « bilingues » (sans traduction simultanée);

3) enfin, les instruments délibératifs exclusivement en français ou exclusivement en anglais, où participent exclusivement les unilingues francophones ou anglophones (sans traduction simultanée).

Le premier dispositif, que nous intitulons « instrument délibératif bilingue », permet à la fois aux unilingues francophones, aux unilingues anglophones et aux citoyens 
«bilingues» de participer à l'exercice démocratique, puisqu'il se complémente d'un service de traduction simultanée (incluant la traduction de tous les documents partagés aux participants et l'animation/facilitation des groupes de discussion dans les deux langues officielles). Ce dispositif a été privilégié par l'État dans la conception et la mise en œuvre de l'exercice sur la réduction de la pauvreté. Bon nombre de facteurs contraignants accompagnent par ailleurs ce dispositif au moment de sa conceptualisation et de sa mise en œuvre. Parmi ceux-ci, les dépenses associées à la «bilinguisation » de l'exercice (traduction simultanée, traduction des documents, embauche d'animateurs bilingues, etc.) et que peuvent difficilement se permettre les organismes communautaires dont la survie dépend exclusivement de subventions gouvernementales. Une autre contrainte est liée à l'inclusion "réelle » des groupes linguistiques minoritaires et au niveau de compréhension de la langue seconde de certains participants. Par exemple, même dans les communautés où le profil linguistique est majoritairement francophone, il existe des citoyens unilingues anglophones qui ont le droit de participer à l'exercice délibératif au même titre que leurs homologues francophones ou «bilingues ». Or, dans de telles situations, les organisateurs privilégient plutôt une démarche exclusivement francophone pour trois raisons : non seulement il s'agit de la langue de la majorité, mais ce choix permet d'économiser des frais substantiels en services de traduction, ainsi qu'en temps. Le faible niveau de littératie dans les communautés francophones est une autre limite à prendre en considération au moment du design des instruments délibératifs dans la mesure où, tel que le soulignent certains auteurs (Avritzer, 2002; Fung, 2003; Habermas, 1991, 1996), les groupes marginalisés ne maîtrisent pas forcément le jargon utilisé. Au Nouveau-Brunswick, près de $66 \%$ de la population francophone ne détient qu'un niveau 2 d'alphabétisme, ce qui veut dire que la capacité de compréhension orale et écrite n'est pas suffisante pour comprendre un rapport gouvernemental, ou tout autre document pouvant servir à informer les participants lors de l'exercice délibératif. Enfin, le dernier facteur contraignant se rapporte à la compréhension limitée de l'enjeu public $\mathrm{du}$ point de vue des deux profils linguistiques. En ce sens, nous avons remarqué que l'enjeu public est défini en fonction des propos de la majorité linguistique (selon ses intérêts, besoins et perceptions), et laisse de côté les particularités de la problématique associées à la minorité linguistique.

Le deuxième dispositif délibératif à l'étude se distingue, quant à lui, par son statut de dispositif "exclusivement en français ou exclusivement en anglais ", mais auquel peuvent par ailleurs participer les citoyens qui sont «bilingues » (dont la langue maternelle n'est pas forcément celle de la langue de la tenue de l'exercice, mais qui maîtrisent néanmoins la langue seconde). Lors de ces exercices, il n'y a aucun service de traduction. C'est ce mécanisme délibératif qui a été privilégié par les représentants de groupes communautaires dans le cas sur la politique familiale. L'obtention du financement pour 
la tenue de l'exercice était conditionnelle au respect de certaines exigences gouvernementales par les acteurs non gouvernementaux, dont celle de prévoir un dispositif qui permettrait aux deux communautés linguistiques de participer à l'exercice. Il s'agissait d'un impératif en vue de recueillir la perspective "bilingue » (des deux communautés linguistiques officielles) de la population néo-brunswickoise à l'égard de l'enjeu public donné. Bien que ce dispositif particulier réponde sur papier aux exigences gouvernementales en termes de bilinguisme, il pose un certain défi au niveau de la représentation symbolique des communautés linguistiques. En effet, tel que nous l'avons constaté dans l'étude du cas sur la politique familiale, très peu de participants à l'exercice partageaient leurs points de vue (intérêts et besoins) selon un angle linguistique anglophone et/ou bilingue. La majorité, pour ne pas dire la totalité, des données recueillies lors de la démarche délibérative brosse un portrait plutôt «francophone » de la réalité entourant les défis, besoins et mesures à prendre en vue d'améliorer le mieuxêtre des familles du Nouveau-Brunswick.

Dans les faits, nous remarquons que les représentants des groupes communautaires à l'origine de l'exercice sur la politique familiale ont déployé, au moment de la mise en œuvre de l'exercice, un troisième type d'instrument délibératif. Celui-ci se distingue par son caractère "exclusivement en français ou exclusivement en anglais, où participent exclusivement les unilingues francophones ou anglophones » (dont la langue maternelle est la même que la tenue de l'exercice délibératif). Tout comme le dispositif précédent, aucun service de traduction n'accompagne ce type d'instrument. Contraints par l'obligation de respecter la vocation «francophone » de leur mandat, les représentants de ces groupes communautaires ont opté pour la mise en œuvre d'un dispositif légèrement différent de ce qu'ils avaient pourtant initialement conçu (en réponse aux exigences gouvernementales pour obtenir les subventions financières). Bien que ce dispositif favorise largement l'expression active d'une communauté linguistique en situation minoritaire, elle élimine totalement la « voix » de l'autre profil linguistique sur le plan du processus de formulation des politiques publiques.

\subsection{La langue comme ressource ou contrainte des groupes communautaires}

Dans un premier temps, nous notons que l'influence de la dimension linguistique sur les groupes/réseaux impliqués (acteur non gouvernemental) a une portée contraignante sur les structures qui se différencient par leur statut linguistique exclusivement francophone, en particulier. L'ambiguïté pour ces groupes se traduit notamment par l'obligation de devoir répondre aux prérogatives de la LLO, tout en respectant leurs mandat et vision d'organisme "francophone " (lesquels concernent plutôt l'avancement des intérêts, besoins et droits des communautés en contexte minoritaire francophone). Ainsi, la composante linguistique peut favorablement avantager les groupes/réseaux à portée 
provinciale qui se distinguent par leur désignation bilingue (notamment en termes d'influence sur le processus de formulation des politiques publiques). En tant que porteparole des deux communautés linguistiques officielles de la province à la fois, ces derniers réussissent mieux à représenter les intérêts, besoins et droits de l'ensemble de la population néo-brunswickoise. Comme nous l'avons remarqué, cette particularité contribue à leur apporter une plus grande crédibilité lors du processus d'élaboration des politiques publiques. Par ailleurs, la question linguistique peut s'avérer contraignante lorsque les groupes/réseaux à portée provinciale se caractérisent par leur désignation unilingue francophone. Le cas sur la politique familiale (l'implication directe du RPEFNB et de l'AFPNB) illustre bien ce constat.

Notre étude fait ressortir que la structure des groupes/réseaux francophones (Marsh et Smith, 2000; Rhodes, 2006; Rhodes et Marsh, 1992), mais aussi leur mode de gouvernance, peuvent avoir un impact (majoritairement contraignant) sur leur degré d'influence lors $\mathrm{du}$ processus de formulation des politiques publiques. Les travaux pionniers de Cardinal et ses collaborateurs (Cardinal et Hudon, 2001; Cardinal et Juillet, 2002; Cardinal, Lang et Sauvé, 2008) et de plusieurs autres auteurs (Forgues, 2012, 2014; Chouinard, 2012) ont permis d'analyser les configurations des mécanismes de gouvernance implantés dans certains programmes gouvernementaux (notamment dans les ententes Canadacommunautés et le Réseau de développement économique et d'employabilité). Ces ancrages théoriques abordent également les enjeux de légitimité et le type de rationalité qui sous-tendent les pratiques de gouvernance en contexte francophone minoritaire. L'une des particularités des communautés vivant en situation minoritaire est qu'elles ne possèdent pas leur propre institution étatique chargée d'assurer leur développement et la conduite de leurs affaires (Forgues, 2014). Ce faisant, les projets qu'entreprennent les groupes/réseaux francophones sont généralement réalisés grâce au soutien des ministères, fédéraux ou provinciaux, concernés. Il convient de souligner que les modalités de gouvernance fondées sur ce type de partenariat avec l'État ne semblent pas mobiliser les communautés francophones (Landry et coll., 2010) et peut même mener à des rapports de pouvoir asymétriques.

De plus, en tant que principal (voire unique) bailleur de fonds, l'État est bien placé pour prescrire aux organismes francophones concernés les conditions à respecter pour bénéficier du financement prescrit. Dans un tel contexte, les projets doivent s'inscrire dans les priorités établies par le gouvernement. Selon Forgues (2015), l'autorité gouvernementale se retrouve en parfaite position pour influencer à sa guise l'orientation du « développement communautaire », en choisissant de soutenir financièrement ou non certains projets, groupes/réseaux ou secteurs d'activités. Par conséquent, les rapports de force entre représentants d'organismes et représentants de l'État parviennent 
difficilement à sortir d'une culture de gestion verticale de l'action (Cardinal et Hudon, 2001). D'autres auteurs soutiennent que cette forme de gouvernance permet au gouvernement qui finance les organismes communautaires en situation minoritaire d'agir envers ceux-ci «comme s'ils constituaient une extension de sa propre fonction publique » (Landry et coll., 2010, p.111). Les risques d'une telle asymétrie peuvent se matérialiser à deux niveaux: dans la structure des organismes communautaires (et notamment dans leur représentation, qui tend à se mouler aux critères des enveloppes budgétaires octroyées par l'État) et dans l'instrumentalisation de ces derniers par l'État (qui en fait des organismes prestataires des services publics).

Nous avons ainsi constaté que les groupes et réseaux provinciaux qui se distinguent par leur statut linguistique «bilingue » possèdent un avantage stratégique au moment de l'ingénierie des mécanismes délibératifs et, plus largement, lors du processus d'élaboration des politiques sociales au Nouveau-Brunswick. En contrepartie, nous avons observé que la composante "francophone » qui caractérise certains organismes provinciaux s'avère un facteur plutôt contraignant à ces chapitres. Ce constat s'explique par la plus grande capacité des groupes bilingues à se faire reconnaître en tant que porteparole des intérêts et besoins des deux communautés linguistiques officielles à l'échelle provinciale (un élément essentiel pour l'élaboration des politiques publiques dans une province officiellement bilingue comme le Nouveau-Brunswick), alors que les organismes francophones sont contraints par un mandat limitatif sur ce plan (représentation des intérêts et besoins des francophones « exclusivement »).

Bien que cette étude ne soit pas centrée sur l'analyse des structures de groupes communautaires à caractère exclusivement anglophone en contexte minoritaire, nous posons l'hypothèse que ces derniers rencontrent fort probablement les mêmes défis que leurs homologues francophones en situation minoritaire. Ce constat est d'autant plus vrai lorsqu'il n'existe pas d'organismes équivalents au sein de l'autre communauté linguistique officielle avec qui partager des affinités convergentes. Par exemple, dans le cas du RPEFNB et de l'AFPNB, il était difficile pour les membres d'établir un dialogue soutenu avec des intervenants du secteur anglophone, puisqu' aucun groupe anglophone équivalent n'existait dans les secteurs d'intervention reliés à la petite enfance et au mieuxêtre de la famille.

De nombreux écrits soulignent que la gouvernance repose à la fois sur la collaboration entre divers acteurs, qui eux partagent à leur tour " pouvoir, ressources et risques en vue d'atteindre des objectifs communs" (Forgues, 2014, p.7). C'est dans ce contexte de nouvelle gouvernance que la gestion horizontale, les pratiques de collaborations intersectorielles et interministérielles, la prolifération des réseaux et les démarches 
décisionnelles décentralisées (comme celles que suppose la mise en cuvre des instruments) se multiplient (Bourgault, 2002; Burlone et coll., 2008; Callon et coll., 2001; Peters, 1998). Selon Cardinal et Hudon (2001), cette nouvelle façon de gouverner contribue à l'accroissement de la complexité dans la coordination efficace de l'action, les groupes et réseaux francophones n'y faisant pas exception. Pour ces auteures, ces pratiques contemporaines posent d'importants défis, notamment en ce qui concerne l'établissement de «nouvelles formes d'imputabilité collective ». Ce constat est d'autant plus problématique lorsque certains acteurs clés du processus décisionnel sont absents et ne participent pas activement à l'exercice démocratique en question.

L'asymétrie entre l'État et les organismes francophones se manifeste également sur le plan de la représentation, qui tend à épouser les critères assignés pour l'obtention des enveloppes budgétaires des gouvernements (provincial et fédéral). En ce sens, la dépendance financière des organismes francophones est une autre caractéristique contraignante au niveau de l'ingénierie des instruments délibératifs et du processus de formulation des politiques publiques. En l'occurrence, soit les organismes francophones modifient leurs pratiques de gouvernance pour que celles-ci coïncident plus directement avec les critères financiers exigés par l'État, soit les groupes et réseaux francophones n'osent pas toujours exprimer ouvertement leurs revendications auprès des participants à un exercice délibératif, et particulièrement auprès de la main qui les nourrit, c'est-à-dire l'État. Dans un contexte de partenariat asymétrique avec l'État, ces exigences systémiques tendent également à limiter le pouvoir d'autonomie des organismes francophones sur le plan de la gestion des activités. Enfin, le gouvernement favorise généralement les modèles de gestion qui sont conçus à partir des assises de la nouvelle gestion publique (Emery, 2005). Dans cette optique de redéfinition de la performance organisationnelle, les échéanciers concernant la mise en œuvre des projets doivent être fidèlement respectés et tous les changements qui dérivent de la planification originale doivent être justifiés auprès du bailleur de fonds. Dans le cas de la politique familiale, par exemple, les obstacles posés par l'inflexibilité des échéanciers et les ressources limitées octroyées par l'État ont grandement influencé l'ingénierie de l'instrument délibératif, sa mise en œuvre et le processus de formulation de la politique familiale (qui n'a finalement jamais vu le jour).

\subsection{Les langues de l'État}

La cohabitation des membres communautaires et étatiques au sein d'un même réseau francophone est plutôt difficile, notamment à cause de l'influence de la variable linguistique sur les institutions étatiques (leurs structures et pratiques de gouvernance). À partir de l'étude du RPEFNB, nous avons constaté que les représentants gouvernementaux, en raison de leur composition et leur mandat, ne sont pas membres 
du réseau au même titre que les organismes francophones. Certains ministères, comme le ministère des Affaires intergouvernementales, sont entre autres responsables de la gestion d'un portefeuille destiné à l'épanouissement et à la vitalité des communautés francophones en contexte minoritaire. D'autres, comme le ministère du Développement social, sont responsables de la gestion et de l'élaboration de politiques publiques qui concernent les deux communautés linguistiques officielles afin de respecter les prérogatives de la LLO. Enfin, certains ministères, comme celui de l'Éducation et du Développement de la petite enfance, reproduisent la dualité linguistique au sens de la LLO et possèdent deux structures de gouvernance éducative indépendantes: une structure pour le secteur anglophone et une autre pour le secteur francophone. La diversité des structures étatiques conditionne incontestablement les pratiques de gouvernance des organismes francophones et bilingues et leur place dans le processus d'élaboration des politiques publiques.

De manière générale, les acteurs gouvernementaux (et même les acteurs non gouvernementaux «bilingues ») conservent plutôt une distance par rapport aux acteurs non gouvernementaux francophones. Au lieu de prendre la forme d'un partenariat, la dynamique observée entre les représentants gouvernementaux et les organismes francophones s'apparente à un exercice de gestion axée sur les résultats, pour s'assurer que les actions privilégiées par le réseau sont conformes aux objectifs des ministères concernés (membres du réseau) et aux critères de financement prescrits. De plus, cette réalité s'est transposée lors de l'exercice délibératif sur la politique familiale. Si la variable linguistique influence grandement les structures étatiques et le processus de production des politiques publiques dans un contexte de rapports linguistiques complexes, elle se manifeste également dans le design du dispositif. Ainsi, un instrument qui n'est pas adapté à ces réalités contextuelles risque de ne pas favoriser de réelles délibérations entre acteurs gouvernementaux et non gouvernementaux (et entre les communautés francophones et anglophones), ce qui peut se traduire par d'importants écueils au niveau $\mathrm{du}$ processus de formulation des politiques publiques. Dans le cas de la politique familiale, les informations recueillies lors de l'exercice délibératif ne représentaient pas adéquatement les besoins et la réalité des deux communautés linguistiques, ce qui a eu pour effet de limiter la portée de la politique publique et de son processus de formulation. 


\subsection{Influence de la variable linguistique sur le processus de formulation des politiques publiques}

L'influence de la variable linguistique sur les institutions étatiques et le processus de production des politiques publiques s'explique également par la LLO du NouveauBrunswick et la Charte canadienne des droits et libertés. Ces deux cadres législatifs régissent, entre autres, les droits fondamentaux des communautés linguistiques en contexte minoritaire d'un territoire donné. Nous avons constaté que l'exercice particulier de restructuration ministérielle dans les domaines de l'éducation et de la petite enfance (qui a donné lieu à la création du ministère de l'Éducation et du Développement de la petite enfance) a causé une certaine ambiguïté chez les acteurs gouvernementaux et non gouvernementaux. Cette observation est particulièrement vraie pour les intervenants communautaires de la petite enfance. La raison de cette confusion réside dans la conjoncture linguistique. Plusieurs acteurs se demandaient si le principe de la «dualité linguistique » devait s'appliquer ou non au secteur du développement de la petite enfance, au même titre que le reste du secteur de l'Éducation. La réponse ne faisait pas l'unanimité au sein des acteurs concernés. Lorsqu'il y a fusion entre des ministères qui n'ont pas les mêmes obligations en matière de dualité linguistique, la formulation des politiques publiques est touchée par cette ambiguïté. Par exemple, doit-on avoir une politique familiale «bilingue» ou bien privilégier l'adoption de deux politiques familiales distinctes adaptées à la réalité linguistique de chacune des communautés linguistiques officielles ? Les mêmes interrogations se posent pour l'enjeu de la pauvreté.

Nos résultats de recherche indiquent que certains ministères sont responsables de la gestion et de la coordination de programmes et services qui concernent exclusivement les communautés linguistiques en contexte minoritaire. Quelques exemples ont été évoqués lors de nos entretiens au sujet de programmes de subventions offerts à des groupes communautaires francophones (comme dans le cas du financement de l'exercice délibératif sur l'élaboration de la politique familiale). Dans ce contexte, l'ambivalence tient au fait que les subventions gouvernementales servent à financer des projets qui, d'une façon ou d'une autre, permettent l'épanouissement des communautés linguistiques francophones (les demandes de financement proviennent fréquemment, pour ne pas dire exclusivement, des groupes communautaires francophones), alors que les exigences gouvernementales en matière d'élaboration de politiques publiques stipulent que celles-ci doivent répondre aux besoins, intérêts et droits des deux communautés linguistiques officielles de la province, conformément à la LLO. L'incidence de la variable linguistique sur le processus de formulation des politiques publiques se traduit donc par une conciliation difficile entre les objectifs et la finalité des mécanismes délibératifs conçus par l'État et ceux mis en place par les acteurs non gouvernementaux. 


\section{Conclusion}

Parmi nos principaux constats, nous avons relevé les effets de la dimension linguistique sur la conception et la mise en œuvre des instruments délibératifs. Cet élément s'ajoute au modèle conceptuel original proposé par Abelson et Gauvin (2006) et aux diverses typologies d'instruments qui existent à ce jour. En plus de mettre en lumière et d'expliquer l'influence d'une conjoncture linguistique complexe comme celle du Nouveau-Brunswick sur l'ingénierie des mécanismes délibératifs, nos constats de recherche permettent $\mathrm{d}$ 'affiner notre compréhension de la dynamique du changement qui s'opère lors d'une démarche de production de politiques sociales dans le contexte bien particulier de la démocratie délibérative.

Le cas du Nouveau-Brunswick s'avère non seulement une source de connaissances pour mieux comprendre la réalité provinciale, mais ouvre un agenda de recherche pour l'échelon infranational bilingue tel que l'Acadie ou d'autres juridictions internationales où cohabitent diverses communautés linguistiques. Dans le contexte de «nouvelle gouvernance» qui caractérise nos administrations publiques contemporaines, nous pouvons supposer que les dispositifs délibératifs sont appelés à se développer et à s'institutionnaliser davantage au cours des prochaines années. Ainsi, la présente étude permet de démontrer que la prise en considération des facteurs contextuels, et notamment la dimension linguistique, devient une condition essentielle pour le succès de l'exercice démocratique en cause.

\section{Bibliographie}

Abelson, J., Gauvin F.-P. (2006). Assessing the Impacts of Public Participation: Concepts, Evidence, and Policy Implications. Canadian Policy Research Networks. 52p.

Abelson, J., Montesani, S., LI, K., Gauvin, F.-P., Martin, E. (2010). Effective Strategies for Interactive Public Engagement in the Development of Healthcare Policies and Programs. Canadian Health Services Research Foundation. Ottawa (Ontario). 49p.

Arnstein, S. (1969). A Ladder of Citizen Participation, Journal of the American Institute of Planners. 35(4), 216-224.

Avritzer, L. (2002). Democracy and the Public Space in Latin America. NJ: Princeton University Press. 208p.

Balch, G. I. (1980). The Stick, the Carrot and Other Strategies: A Theoretical Analysis of Governmental Intervention. Law and Policy Quarterly, 2(1), 35-60. 
Beierle, T. C., Cayfor, J. (2002). Democracy in Practice: Public Participation in Environmental Decisions, Washington (DC): Resources for the Future. 160p.

Bemelmans-Videc, M.-L., Rist, R. C., Vedung, E. O. (Dirs.). (2003) Carrots, Sticks, and Sermons. Policy Instruments and Their Evaluation. Comparative Policy Evaluation. 7(1). Transaction Publishers, 280p.

Beste, S. (2013). Contemporary Trends of Deliberative Research: Synthesizing A New Study Agenda. Journal of Public Deliberation, 9(2), 1-44.

Bingham, L. B., Nabatchi, T., O'Leary, R. (2005). The New Governance: Practices and Processes for Stakeholder and Citizen Participation in the Work of Government. Public Administration Review, 65(1), 547-558.

Bishop, P., Davis, G. (2002). Mapping public participation in policy choices. Australian Journal of Public Administration, 61(1), 14-29.

Black, L. W., Thomas, N. L., Shaffer, T. J. (2014). The State of Our Field: Introduction to the Special Issue. Journal of Public Deliberation, 10(1), art. 1, 1-5.

Blondiaux, L. (2004). Prendre au sérieux l'idéal délibératif : Un programme de recherche. Swiss Political Science Review, 10(4), 158-169.

Blondiaux, L. (2008). Le nouvel esprit de la démocratie : Actualité de la démocratie participative. France : Éditions du Seuil et La République des Idées. 112p.

Bourgault, J. (Dir.) (2002). Horizontalité et gestion publique. Collection Management public et gouvernance. Québec: Les Presses de l’Université Laval. 354p.

Bresser, H. TH. A., O'Toole Jr., L. J. (1998). The Selection of Policy Instruments: A Network-Based Perspective. Journal of Public Policy, 18(3), 213-239.

Briggs, X. (2008). Democracy as Problem Solving: Civic Capacity in Communities Across the Globe. Cambridge (MA): MIT Press. 388p.

Bryson, J. M., Quick, K. S., Schively Slotterback, C., Crosby, B. C. (2013). Designing Public Participation Processes. Public Administration Review, 73(1), 23-34.

Burlone, N., Andrew, C., Chiasson, G., Harvey, J. (2008). Horizontalité et gouvernance décentralisée: les conditions de collaboration dans le contexte de l'action communautaire. Administration publique du Canada, 51(1), 127-142.

Callon, M., Lascoumes, P., Barthe, Y. (2001). Agir dans un monde incertain. Essai sur la démocratie technique. Paris : Seuil. 357p. 
Carcasson, M. (2009). Occasional Paper 2-Beginning with the End in Mind: A Call for Goal-Driven Deliberative Practice. Public Agenda/Centre for Advances in Public Engagement. New York. 16p.

Cardinal, L., Juillet, L. (2002). L'Ontario francophone et la gouvernance des minorités de langue officielle au Canada : une étude préliminaire. Ottawa (Ontario), Comité de direction de l'Entente Canada-communauté Ontario. 34p.

Cardinal, L., Hudon, M.-È. (2001). La gouvernance des minorités de langue officielle au Canada: une étude préliminaire. Commissariat aux langues officielles, Ottawa (Ontario). Repéré à http://www.clo-ocol.gc.ca/html/stu etu 112001 f.php

Cardinal, L., Lang, S., Sauvé, A. (2008). Les minorités francophones hors Québec et la gouvernance des langues officielles : portrait et enjeux. Francophonies d'Amérique, (26), 209-233.

Chouinard, S. (2012). Quel avenir pour le projet autonomiste des communautés francophones en situation minoritaire? Minorités linguistiques et société, (1), 195213.

Einsiedel, E. (2002). Assessing a controversial medical technology: Canadian public consultation on xenotransplantation. Public Understanding of Science, 11(4), 315-331.

Eliadis, P., Hill, M. M., Howlett, M. (Dirs.). (2005), Designing Government: From Instruments to Governance. McGill-Queen's University Press, 528p.

Emery, Y. (2005). La gestion par les résultats dans les organisations publiques : de l'idée aux défis de la réalisation. Télescope, 12(3), 1-11.

Fishkin, J. (2009). When the People Speak. Deliberative Democracy and Public Consultation. New York: Oxford University Press. 256p.

Forgues, É. (2012). Le partenariat des communautés francophones en situation minoritaire avec l'État : frein ou tremplin à l'autonomie ? Minorités linguistiques et société, (1), 180-194.

Forgues, É. (2014). La gouvernance de la communauté acadienne au NouveauBrunswick. Dans Oueslati, S., Magord, A. (Dirs.). Qui gouverne aux États-Unis et au Canada ? Cahiers du MIMMOC. (11), 23p.

Forgues, É. (2015). Ni verticale, ni horizontale : la gouvernance communautaire au sein de la francophonie en situation minoritaire au Canada. Revue gouvernance. 25p.

Fung, A. (2003). Associations and Democracy: Between Theories, Hopes, and Realities. Annual Reviews of Sociology, 29, 515-539. 
Gauthier, M., Simard, L., Waaub, J.-P. (2011). Public participation in strategic environmental assessment (SEA): Critical review and the Quebec (Canada) approach. Environmental Impact Assessment Review, 31(1), 48-60.

Goodin, R. E. (2012). How Can Deliberative Democracy Get a Grip? The Political Quarterly, 83(4), 806-811.

Gudowsky, N., Bechtold, U. (2013). The Role of Information in Public Participation. Journal of Public Deliberation, 9(1), 1-35.

Habermas, J. (1991). The Structural Transformation of the Public Sphere: An Inquiry into a Category of Bourgeois Society (traduit par T. Burger). Cambridge: The MIT Press. 326p.

Habermas, J. (1996). Between Facts and Norms: Contributions to a Discourse Theory of Law and Democracy (traduit par W. Rehg). Cambridge: The MIT Press. 675p.

Hendricks, C. M. (2008). The Social Context of Public Deliberation: Letting Practice Shape Theory. International Journal of Public Participation, 2(1), 87-91.

Hood, C. (1983). The Tools of Government. Public Policy and Politics Series. London: Macmillan. 178p.

Hood, C. (2006). Chapter 22-The Tools of Government in the Information Age. Dans M. Moran, M. Rein et R. E. Goodin (Dirs.). (2006). The Oxford Handbook of Public Policy. Oxford University Press. 469-481.

Hood, C. (2007). Intellectual Obsolescence and Intellectual Makeovers: Reflections on the tools of Government After Two Decades. Governance, 20(1), 127-144.

Howlett, M. (2005). Chapter 2-What Is a Policy Instrument? Policy Tools, Policy Mixes, and Policy-Implementation Styles. Dans Eliadis, P., Hill, M. M., Howlett, M. (Dirs.). (2005). Designing Government: From Instruments to Governance. McGillQueen's University Press. 31-50.

Howlett, M. (2011). Designing Public Policies. Principles and instruments. Routledge, Oxon. 236p.

Howlett, M. (2014). Chapter 9-Policy Design. What, Who, How and Why? Dans Halpern, C., Lascoumes, P., Le Galès, P. (Dirs.). (2014). L'instrumentation de l'action publique. Controverses, résistance, effets. Presses de Sciences Po, 281-316.

Howlett, M., Ramesh, M. (2003). Studying Public Policy: Policy Cycles and Policy Subsystems. Oxford University Press. 311p.

Howlett, M., Ramesh, M., Perl, A. (2009). Studying Public Policy Cycles and Subsystems. Third Edition. Toronto (Ontario): Oxford University Press. 336p.

Revue Gouvernance

Volume 14, numéro 1, 2017 
Korthals, M. (2011). Deliberations on the Life Sciences: Pitfalls, Challenges and Solutions. Journal of Public Deliberation, 7(1), article 8, 13p.

Landry, R., Forgues, É., Traisnel, C. (2010). Autonomie culturelle, gouvernance et communautés francophones en situation minoritaire au Canada. Politique et société, 29(1), 91-114.

Lee, C. W. (2011). Five Assumptions Academics Make About Public Deliberation, And Why They Deserve Rethinking. Journal of Public Deliberation, 7(1), art. 7, 48p.

Linder, S. H., Peters, B. G. (1989). Instruments of Government: Perceptions and Contexts. Journal of Public Policy, 9(1), 35-58.

Lowi, T. J. (1966). Distribution, Regulation, Redistribution: The Functions of Government. Dans Ripley, R. B. (Dir.). (1966). Public Policies and Their Politics: Techniques of Government Control. New York: W. W. Norton, 27-40.

Lowi, T. J. (1985). The State in Politics: The Relation between Policy and Administration. Dans Noll, R. G. (Dir.). (1985). Regulatory Policy and the Social Sciences. Berkeley: University of California Press, 67-105.

Marsh, D., Smith M. (2000). Understanding Policy Networks: towards a Dialectical Approach. Political Studies, 48(1), 4-21.

Mc Cormack, B., Kitson, A., Harvey, G., Rycroft-Malone, J., Titchen, A., Seers, K. (2002). Getting evidence into practice: the meaning of 'context'. Journal of Advanced Nursing, 38(1), 94-104.

Mitton, C., Smith, N., Peacock, S., Evoy, B., Abelson, J. (2009). Public participation in health care priority setting: A scoping review. Health Policy, 91(3), 219-228.

Nabatchi, T. (2010). Addressing the Citizenship and Democratic Deficits: Exploring the Potential of Deliberative Democracy. American Review of Public Administration. 40(4), 376-399.

Nabatchi, T., Amsler, L. B. (2014). Direct Public Engagement in Local Government. American Review of Public Administration, 44(4(S)), 63S-88S.

O'Faircheallaigh, C. (2010). Public participation and environmental impact assessment: purposes, implications, and lessons for public policy making. Environmental Impact Assessment Review, 30(1), 19-27.

O'Meally, S. C. (2013). Mapping Context for Social Accountability: A Resource Paper. Social Development Department. World Bank, Washington (D.C.), 95p.

OCDE (2001). Des citoyens partenaires. Paris (France). 291p.

Revue Gouvernance

Volume 14, numéro 1, 2017
40 
Perote-Pena, J., Piggins, A. (2015). A Model of Deliberative and Aggregative Democracy. Economics and Philosophy, 31(1), 93-121.

Peters, B. G. (1998). La gestion d'un gouvernement horizontal : l'aspect de la coordination. Centre canadien de gestion. Ottawa (Ontario). 75p.

Prades, J.-L. (2006). Intervention et communication dans un dispositif participatif : les cas limites: enfants, personnes âgées et personnes handicapées. Nouvelles pratiques sociales, 18(2), 110-126.

Rhodes, R. A. W. (2006). Policy Network Analysis. Dans Moran, M., Rein, M., Goodin, R. R. (Dirs.). (2006). The Oxford Handbook of Public Policy, 425-447.

Rhodes, R. A. W., Marsh, D. (1992). New Direction in the Study of Policy Networks. European Journal of Political Research, 21(1-2), 181-205.

Rowe, G., Frewer, L. J. (2000). Public participation methods: A framework for evaluation. Science, Technology, \& Human Values, 25(1), 3-29.

Rowe, G., Marsh, R., Frewer, L. J. (2004). Evaluation of a Deliberative Conference. Science, Technology \& Human Values, 29(1), 88-121.

Ryfe, D. M. (2005). Does Deliberative Democracy Work? Annual Review of Political Science, 8, 49-71.

Ryfe, D. M. (2007). Toward a Sociology of Deliberation. Journal of Public Deliberation, 3(2), $1-27$.

Salamon, L. M., Lund, M. S. (1989). Chapter 2: The Tools Approach: Basic Analytics. Dans Salamon, L. M. (Dir.). (1989). Beyond Privatization: The Tools of Government Action. Washington: Urban Institute, 23-50.

Sanders, L. M. (1997). Against Deliberation. Political Theory, 25(3), 347-376.

Santo, C., Ferguson, N., Trippel, A. (2010). Engaging Urban Youth Through Technology: The Youth Neighborhood Mapping Initiative. Journal of Planning Education and Research, 30(1), 52-65.

Schneider, A., Ingram, H. (1990). Behavioral Propositions of Policy Tools. Journal of Politics, 52(2), 510-529.

Sheedy, A., Mackinnon, M. P., Pitre, S., Watling, J. (2008). Handbook on Citizen Engagement: Beyond Consultation. Canadian Policy Research Networks. 54p.

Shipley, R., Utz, S. (2012). Making it Count: A Review of the Value and Techniques for Public Consultation. Journal of Planning Literature, 27(1), 22-42. 
Sintomer, Y. (2011). Délibération et participation: Affinité élective ou concepts en tension ? Participations, 1(1), 239-276.

Steiner, J. (2012). The Foundations of Deliberative Democracy. Empirical Research and Normative Implications. Cambridge: Cambridge University Press. 228p.

Tellier, G. (2014). L'usage des consultations prébudgétaires au Canada : étude de cas de la Colombie-Britannique. Dans Djouldem, M., Tellier, G., de Visscher, C. (Dirs.). (2014). Les réformes des finances publiques. Enjeux politiques et gestionnaires. Paris: Bruylant, 83-107.

Thurston, W. E., Mackean, G., Vollman, A., Casebeer, A., Weber, M., Maloff, B., Bader, J. (2005). Public participation in regional health policy: a theoretical framework. Health Policy, 73(3), 237-252.

Varone, F. (1998). Le choix des instruments des politiques publiques : une analyse comparée des politiques d'efficience énergétique du Canada, du Danemark, des États-Unis, de la Suède et de la Suisse. Bern : Haupt. 370p.

Venne, M. (2011). Des citoyens responsables. Télescope, 17(1), 194-212.

Yankelovich, D. (2001). The Magic of Dialogue, Transforming conflict into cooperation. New York: First Touchstone. 240p.

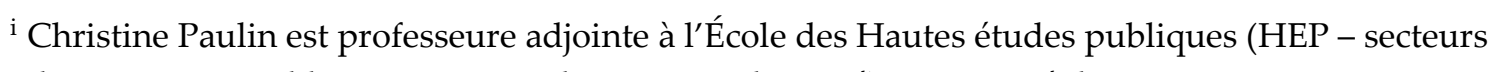
administration publique et gestion des services de santé), Université de Moncton 